\title{
Transfert de gènes dans les cellules hématopoïétiques: obscur objet du désir de voir et de manipuler la vraie cellule souche?
}

L'expression d'une nouvelle information génétique dans les cellules souches, progéniteurs ou cellules différenciées du tissu hématopoïétique, est l'un des enjeux majeurs de la thérapie génique. Bien que de nombreuses étapes techniques restent à maîtriser, cette approche ouvre de nombreuses possibilités pour le traitement des maladies héréditaires (déficits immunitaires, déficits enzymatiques, anomalies de l'hémoglobine) et acquises. La pratique de la transplantation des cellules souches $\left(\mathrm{CD} 34^{+}\right)$chez des malades atteints de cancers ou d'hémopathies permet d'envisager la modification génétique de ces cellules ou de leur progénie, soit pour suivre la reconstitution hématologique (gene marking), soit pour sélectionner in vivo une population hématologique non atteinte ou résistante à un processus de leucémogenèse ou d'oncogenèse (gène $M D R$ de résistance à divers médicaments utilisés en chimiothérapie, anti-oncogènes...) [1]. Enfin, s'il s'avérait qu'à partir des cellules souches du sang périphérique, ou du sang de cordon, on puisse reconstituer l'ensemble des lignages, dont le lignage lymphoïde ou celui des cellules dendritiques, ce qui est de plus en plus probable, des perspectives très importantes s'ouvriraient pour le traitement de l'infection par le VIH [2].

La seconde réunion de l'Action concertée sur la «Manipulation génétique des cellules souches hématopoïétiques » s'est déroulée à Marseille les 17 et 18 octobre 1995 . Dix-sept laboratoires européens y ont participé, montrant ainsi l'intérêt

tifique européenne pour ce domaine.

\section{Des gènes marqueurs aux gènes thérapeutiques}

L'ensemble des participants a manifesté la volonté d'utiliser des approches de marquage avant d'aborder le problème de transfert génique en vue d'une action thérapeutique. Le gène marqueur neoR, très controversé, a fait place dans de nombreuses équipes au gène $L a c Z$ et à ses dérivés [3], utilisés dans les cellules CD34+ (Bagnis, Marseille, France ; Louache, Villejuif, France), ou les lymphocytes T (Imbert, Marseille); un protocole clinique de marquage des progéniteurs hématopoiétiques avec le gène nls $L a c Z$ comportant le signal de localisation nucléaire nls est en préparation (Mannoni, Marseille). D'autres marqueurs ont été proposés, comme la glycophorine B (Dubart, Villejuif), ou la protéine GFP (green fluorescent protein) dérivée de la méduse [4] (Rueda, Barcelone, Espagne) ; l'intérêt de la détection membranaire de la glycoprotéine $\mathrm{P}$ codée par le gène $M D R$ [5] a été rappelé, ce qui permettrait de pallier la difficulté d'analyser le transfert génique dans les populations de cellules résistantes au taxol (Ostertag, Hambourg, Allemagne). Plusieurs perspectives thérapeutiques ont cependant été présentées: (1) l'intensification chimiothérapeutique grâce à l'activité du produit du gène $M D R$ (Ostertag; Thierry, Bethesda, MD, USA) et le contrôle de la maladie du greffon contre l'hôte par destruction des cellules $\mathrm{T}$ du donneur par l'action du gène $H S V$-TK $\left(\mathrm{m} / \mathrm{s} n^{\circ} 7\right.$, vol. $8, p .728$; $n^{\circ}$ 6, vol. 11, p. 919) (Tiberghien, Besançon, France) [6] ; (2) les traitements des déficiences génétiques classiques par l'utilisation des gènes codant pour les protéines Phox (NADPH-oxydases) dont la déficience est associée à la granulomatose chronique [7] (Grez, Madrid, Espagne), ou par le gène codant pour l'interféron $\alpha$ pour le traitement de leucémies myéloïdes chroniques (De Verneuil, Bordeaux, France) ; et (3) le traitement de l'infection par le VIH par l'expression inductible (Methali, Strasbourg, France) ou constitutivement faible (Lauret, Orsay, France) de l'interfé$\operatorname{ron} \beta[8,9]$.

\section{Rétrovirus : monopole et évolution}

Les vecteurs rétroviraux restent actuellement les vecteurs les plus utilisés pour transférer de nouveaux gènes dans les cellules hématopoïétiques. Seul un groupe a rapporté l'utilisation de complexes ADN-liposomes (Thierry) dans la transfection de cellules hématopoïétiques murines. Aucun groupe européen n'a présenté de travaux utilisant des vecteurs dérivés des AAV (adeno-associated virus); cela reflète le peu de travaux effectivement réalisés avec ces virus dont les possibilités d'intégration cellulaire et les propriétés ne sont pas encore bien connus [10]. Cette tendance se confirme au niveau des protocoles cliniques en cours de préparation ou réalisés actuellement dans le monde; plus de $70 \%$ des protocoles cliniques de thérapie génique utilisent des vecteurs rétroviraux [11].

Le transfert de gène dans les cellules 
hématopoïétiques est effectué le plus souvent à l'aide de structures rétrovirales défectueuses dérivées du virus de Moloney, Mo-MLV (murine leukemia virus) ; la raison est essentiellement historique puisque c'est ce virus qui a été utilisé pour les premières lignées d'encapsidation et la création des premiers virus défectueux dont dérivent de nombreuses constructions [12]. Si on peut noter parfois une combinaison d'éléments rétroviraux provenant de plusieurs virus, comme le LTR (long terminal repeat) du virus du sarcome myéloprolifératif, MPSV [13], la structure des vecteurs est le plus souvent de facture assez classique: le gène d'intérêt est placé sous le contrôle du promoteur viral localisé dans le LTR ou d'un promoteur interne, souvent associé à un gène de sélection comme le gène neoR. L'archétype de ces constructions est le vecteur BAG exprimant le gène $L a c Z$ sous le contrôle du promoteur viral localisé dans le LTR et le gène neoR sous le contrôle d'un promoteur interne dérivé du SV40 [14]. Seuls quelques groupes ont présenté des travaux concernant l'évolution de cette structure virale. L'expression d'un transgène étant limitée au spectre d'expression du promoteur viral dans les vecteurs classiques, le groupe de W. Ostertag a développé l'étude comparative de vecteurs rétroviraux dérivés d'autres types de rétrovirus murins comme le SFFV (spleen focus forming virus), le MESV (murine erythroleukemia virus), ou le MHSV (murine Harvey sarcoma virus). Leurs résultats montrent que l'utilisation de LTR dérivés du SFFV permet d'obtenir un niveau d'expression élevé du transgène dans certaines lignées hématopoiétiques. Le groupe italien d'Antonio Tabilio (Perugia) a présenté des résultats préliminaires concernant l'utilisation de vecteurs rétroviraux dicistroniques dotés d'une séquence IRES (internal ribosome entry site) [15] ; ils permettent la coexpression de deux gènes transférés dans des cellules hématopoïétiques. Actuellement, l'ensemble des travaux réalisés avec des vecteurs de type IRES s'accordent pour montrer une réelle coexpression des deux gènes, ouvrant ainsi des possibilités de co-transfert de deux activités, ce qui était difficile avec les vecteurs rétroviraux plus classiques (voir plus haut). Le contrôle de l'expression n'a été abordé qu'à propos de nouveaux vecteurs rétroviraux portant des promoteurs inductibles par la protéine Tat du virus VIH ou, dans l'avenir, par le couple de protéines Tat et Rev, étudiés en vue du traitement de l'infection par le VIH (Methali) [8]. Malgré les nombreux éléments qui font soupçonner la quiescence des progéniteurs hématopoïétiques les plus immatures, il est à noter qu'aucun travail important n'a été rapporté, tant dans cette réunion que dans la littérature, sur la conception de vecteurs dérivés de lentivirus qui auraient la capacité d'infecter des cellules quiescentes, encore inaccessibles aux vecteurs rétroviraux murins. L'utilisation de nouveaux types de vecteurs ne pourra se développer qu'après que des analyses comparatives auront été publiées concernant leurs possibilités et leurs limites. La prudence est requise avant de s'engager dans de longs travaux utilisant de nouveaux vecteurs. De plus, la plupart des travaux publiés concernent le comportement des vecteurs dans des lignées cellulaires, ce qui peut parfois (souvent) masquer les difficultés d'utilisation rencontrées avec des cellules primaires.

Il est regrettable que la recherche de promoteurs s'exprimant spécifiquement au cours de l'hématopoïèse ne soit encore que débutante; ils seront très utiles pour concevoir de nouveaux vecteurs s'exprimant spécifiquement à certaines étapes de la différenciation hématopoïétique. Cette recherche a été élégamment illustrée par les travaux de détection de promoteurs inductibles par le GM-CSF utilisant des techniques de gene-trap dans les cellules TF-1*. Les techniques de gene-trap regroupent

\footnotetext{
* lignée de cellules hématopoïétiques humaines immatures dépendante de certains facteurs de croissance, dont le GM-CSF, pour sa prolifération et encore capable de s'engager dans plusieurs voies de différenciation, provenant d'un malade atteint d'erythroleucémie [15].
}

toute tentative de placer dans le génome d'une cellule hôte une séquence codante exprimant une activité de sélection (gène marqueur, gène de résistance à un antibiotique...) sous le contrôle d'un promoteur associé à l'activité que l'on désire étudier (dans ce cas, l'induction par le GM-CSF) et, par ce biais, de piéger, localiser et analyser le gène impliqué.

On peut déplorer aussi le manque de travaux fondamentaux utilisant les outils du transfert de gène dans les progéniteurs hématopoiétiques (pour une exception voir [16]).

La mise au point de nouvelles lignées d'encapsidation dérivées de cellules humaines pour produire les vecteurs rétroviraux a fait l'objet de travaux importants (Cosset, Lyon, France); elles sont apparemment plus efficaces pour transférer des gènes dans les cellules CD34+ (Louache) que les lignées classiques dérivées de cellules NIH3T3. Des lignées cellulaires ont été également modifiées, exprimant à leur surface la forme membranaire du SCF (stem cell factor) ; elles permettent d'infecter les progéniteurs hématopoïétiques résistants au traitement par le 5-fluoro-uracile (Casimir, Londres, Grande-Bretagne) ; il a été proposé également de faire un tri sélectif des lignées productrices capables d'infecter les cellules hématopoïétiques (Bagnis). Une plus grande efficacité d'infection a été observée avec des lignées d'encapsidation produisant des vecteurs qui se fixent temporairement sur le récepteur de l'EGF, avant que le virus n'interagisse avec le récepteur amphotrope (Nilson, Londres, Grande-Bretagne). De plus, la description de lignées productrices de vecteurs moins sensibles au complément (Cosset) permet d'envisager l'évolution future de la thérapie génique ex vivo vers la thérapie génique in vivo .

\section{Cuisine et recettes : transduction et cellules hématopoiétiques}

L'absence de consensus concernant les protocoles de transduction des progéniteurs hématopoïétiques, principalement les populations de cellules exprimant l'antigène $\mathrm{CD} 34^{+}$ou 
les lymphocytes $T$, marque bien le besoin de définir et d'améliorer les conditions de transfert génique. Le choix des cellules hématopoiétiques comme cellules cibles pour la thérapie génique ne semble pas devoir être remis en cause. Les prélèvements de cellules de la moelle osseuse étant en diminution constante, on fait de plus en plus appel aux cellules CD34+ mobilisées dans le sang périphérique par le G-CSF et collectées par cytaphérèse [17], ou aux cellules $\mathrm{CD}^{+} 4^{+}$du sang de cordon ombilical prélevées immédiatement après la naissance $[18,19]$. De nombreux travaux démontrent que des populations $\mathrm{CD}^{+} 4^{+}$co-exprimant l'antigène Thy-1 [20] ou, CD34+/lin- et n'exprimant pas encore l'antigène CD38 [21] représentent des populations de cellules plus immatures au cœur même de la fraction cellulaire exprimant l'antigène CD34 [22]. Ces cellules, qui théoriquement confèrent à la population $\mathrm{CD}^{+} 4^{+}$la capacité de maintenir, voire de reconstituer, l'hématopoïèse à long terme, deviennent ainsi une cible d'intérêt majeur du transfert de gènes. Le transfert du gène marqueur nls $L a c Z$ dans des cellules $\mathrm{CD} 34^{+} / \mathrm{Thy}^{+}$a été présenté (Bagnis). La coculture des cellules productrices de vecteurs et des cellules cibles semble toujours favoriser le transfert de gènes: lorsqu'ils sont cocultivés avec des cellules productrices de vecteurs, $10 \%$ à $50 \%$ des progéniteurs hématopoïétiques expriment le transgène (Bagnis, Louache, Hatzfeld, Villejuif). L'infection des lymphocytes $\mathrm{T}$ (Imbert, Lauret) se révèle encore plus difficile ; $1 \%$ à $10 \%$ des cellules sont infectées par les techniques classiques utilisant les surnageants de cultures contenant des rétrovirus, alors que $50 \%$ à $80 \%$ des lymphocytes sont infectés par les techniques de coculture [23]. Les effets de l'association classique de cytokines, IL3, IL6 et SCF [24] sur la stimulation des progéniteurs hématopoïétiques et, potentiellement, sur leur capacité d'être infectés pourraient éventuellement être améliorés en inhibant le TGFß (Hatzfeld), en utilisant des supports de culture constitués de cellules murines stromales (Charbord, Besançon) caractérisation des cellules cibles: analyse de l'expression des récepteurs de cytokines (Conche Bello-Fernandez, Vienne), analyse de l'expression des récepteurs de virus amphotropes (Bregni, Milan, Italie), et analyse du cycle cellulaire (Lemoine, Paris) au cours de la prolifération/différenciation des cellules cibles. Il semble nécessaire d'induire la division cellulaire tout en maintenant la totipotence des progéniteurs hématopoiétiques dans la reconstitution hématologique. Une approche technique de cet équilibre prolifération/différenciation pourrait être envisagée en utilisant un support stromal autologue, allogénique ou xénogénique, qui permettrait la survie des cellules très immatures et des véritables «cellules souches» [26]. De nombreux travaux sont actuellement en cours, et l'intérêt de ces approches et de leurs éventuelles combinaisons reste à confirmer.

Outre le fait de savoir manipuler la cellule à infecter, il est nécessaire de mettre au point des essais fonctionnels des cellules très immatures. On ne peut que rappeler les difficultés de mise au point des LTC-IC (long term culture-initiating cells) [27], et du modèle SCID humanisé [28] qui permet d'analyser la reconstitution lymphoïde. Des solutions ont été proposées pour pallier ces problèmes avec, par exemple, la détection des colonies HPP-GEMM (high proliferative potential - granulocytes - erythrocyte monocytes - megakaryocyte) dérivant de la prolifération et de la différenciation de cellules très immatures dans un milieu de méthylcellulose (Hatzfeld).

\section{Du laboratoire à la clinique}

La journée éducative des "Ateliers de thérapie cellulaire" organisée par C. Cailliot (Amgen-France) sur le thème du transfert de gène dans les cellules hématopoiétiques qui a suivi le workshop de l'Action concertée européenne était consacrée aux réalisations cliniques. La présentation des travaux cliniques de transfert du gène de l'adénosine désaminase dans les cellules CD $34^{+}$ (Nienhuis, USA), ainsi que du transfert du gène $M D R$ dans les cel- lules CD34 ${ }^{+}$(Hesdorffer, New York, USA) ont fait état des approches préalables à ces traitements, et de l'efficacité de transfert et d'expression des transgènes sans apporter de conclusions thérapeutiques majeures. Les présentations des équipes de Marseille (Bagnis), avec le transfert du gène nlsLacZ dans des cellules CD $34^{+}$dérivées du sang périphérique, de Besançon (Tiberghien) avec la modulation de l'alloréactivité par transfert du gène $H S V-T K$ dans les lymphocytes T, de Rijswÿk (Valerio) avec le transfert du gène codant pour l'ADA, et de Manchester (Spooncer) avec le transfert du gène codant pour l' $\alpha$ L-iduronidase ( $\alpha$ IDUA) dans le traitement de la granulomatose chronique ont représenté les travaux européens les plus proches ou déjà engagés dans la réalisation clinique.

\section{État des lieux et avenir}

Cet état des lieux du transfert de gène dans les cellules hématopoïétiques en Europe est actuellement très contrasté, avec une recherche en amont qui commence à développer des outils mieux adaptés à la thérapie génique, et une réalisation clinique en aval, encore pauvre, et limitée dans le choix de ses outils par la réglementation, mais également par la distance qui sépare souvent le protocole expérimental de l'application clinique. Ainsi, l'étude de la manipulation des cellules hématopoïétiques par le transfert de gènes marqueurs, que ce soit au laboratoire ou en clinique, se révèle clairement fondamentale, et de fait précède l'ensemble des travaux présentant des approches thérapeutiques. Les deux problèmes majeurs manifestes actuellement sont de deux ordres. Le premier est technique et concerne la difficulté de mettre au point des protocoles d'infection efficaces et des vecteurs capables de soutenir une expression à long terme ou une expression contrôlée. Le second concerne les difficultés de caractériser et de manipuler les cellules cibles: la majorité des travaux sont réalisés sur les populations $\mathrm{CD}^{+} 4^{+}$mais on sait déjà que, si ces cellules sont les seules dispo- 
nibles actuellement pour la clinique, la cible d'intérêt majeur est une population encore plus réduite, comme les cellules CD34+/Thy1 $1^{+}$. Enfin, un enjeu d'avenir qui complétera les perspectives de la manipulation génétique des cellules hématopoïétiques est le développement de l'utilisation des progéniteurs hématopoiétiques en tant que vecteurs de nouvelles informations génétiques pour d'autres cellules, et non pas comme cellules cibles de cette information.

Que ce soit en terme de vecteurs, de protocoles de transduction, de cellules cibles, de techniques d'analyse de la transduction, de gènes à transférer, une conclusion majeure qui se dégage de cette réunion est le caractère totalement ouvert sur l'avenir et prospectif de la manipulation génétique des cellules hématopoḯtiques. Après une première vague de résultats très divers, un nouveau mouvement, plus réfléchi, s'amorce dans ce domaine et devrait permettre de progresser, tant sur le plan expérimental que sur le plan clinique. Il reste à souhaiter que le caractère mesuré et progressif de cette seconde vague soit compris par tous les intervenants, scientifiques, médicaux, publics, et industriels, de la thérapie génique.

La prochaine réunion de l'Action concertée européenne aura lieu à Florence (Italie) en 1996 et sera organisée par M. Bregni et A. Tabilio

\section{Claude Bagnis \\ Christian Chabannon \\ Patrice Mannoni}

Centre de thérapie génique, Institut PaoliCalmettes, BP156, 13273 Marseille Cedex 09, France.

\section{TIRÉS À PART}

P. Mannoni.

$m / s n^{\circ} 1$, vol. 12 , janvier 96

\section{RÉFÉRENCES}

1. Heslop HE, Roskrow M. Gene transfer for the therapy of hematologic malignancy. Curr Op Hematol 1995 ; 2 : 417-22.

2. Yu M, Poeschla E, Wong Staal F. Progress towards gene therapy for HIV infection. Gene Ther 1994; 1 : 13-26.

3. Bagnis C, Chabannon C, Mannoni P. Marking human progenitors and T lymphocytes with B-galactosidase genes. Cancer Gene Ther 1996 (sous presse).

4. Prasher DC. Using GFP to see the light. Trends Genet 1995; 11: 320-3.

5. Tiberghien P, Reynolds CW, Keller J, Spence S, Deschaseaux M, Certoux JM, Contassot E, Murphy WI, Lyons R, Chiang Y, Hervé P, Longo DL, Ruscetti FW. Ganciclovir treatment of Herpes simplex thymidine kinase-transduced primary $\mathrm{T}$ lymphocytes: an approach for specific in vivo donor T-cell depletion after bone marrow transplantation. Blood 1994; 84: 1333-41.

6. Sekhsaria S, Galilin JI, Lindon GF, Mallory RM, Mulligan RC, Malech HL. Peripheral blood progenitors as a target for genetic correction of p47phox-deficient chronic granulomatous disease. Proc Natl Acad Sci USA 1993; 90 : 7446-50.

7. Vieillard V, Lauret E, Rousseau V, De Mayer E. Blocking of retroviral infection at a step prior to reverse transcription in cells transformed to conctitutively express interferon $\beta$. Proc Natl Acad Sci USA 1994; 91: 2689-93.

8. Tania Sorg T, Leissner P, Calenda V, Leroy $\mathrm{P}$, Sanhadji K, Touraine JL, Pavirani A,

Mehtali, M. Thérapie génique de maladies infectieuses : le modèle du SIDA. médecine/ sciences $1996 ; 12$ : 13-24.

9. Goodman S, Xiao X, Donahue RE, Moulton A, Miller J, Walsh C, Young NS, Samulski RJ, Nienhuis AW. Recombinant adeno-associated virus-mediated gene transfer into hematopoietic progenitor cells. Blood 1994; 84: 1492-500.

10. Mann R, Mulligan RC, Baltimore D. Construction of a retrovirus packaging mutant and its use to produce helper-free defective retrovirus. Cell 1983; 33: 153-9.

11. Valère $\mathrm{T}$. Thérapie génique: le point sur les essais cliniques. médecine/sciences 1996 ; 12: 73-83.

12. Clapp DW, Freie B, Srour E, Yoder MC, Fortney K, Gerson SL. Myeloproliferative sarcoma virus directed expression of $\beta$-galactosidase following retroviral transduction of murine hematopoietic cells. Exp Hematol 1995; 23: 630-8.

13. Price J, Turner D, Cepko C. Lineage analysis in the vertebrate nervous system by retrovirus-mediated gene transfer. Proc Natl Acad Sci USA 1987; 84: 156-60.

14. Ghattas IR, Sanes JR, Majors JE. The encephalomyocarditis virus internal ribosome entry site allows efficient coexpression of two genes from a recombinant provirus in cultured cells and embryo. Mol Cell Biol 1991; 11: 5848-59.
15. Kitamura T, Tange T, Terasawa T, Chiba S, Kuwaki T, Miyagawa K, Piao Y, Miyazono K, Urabe A, Takaku F. Establishment and characterization of a unique human cell line that proliferates dependently on GM-CSF, IL-3, or erythropoietin. J Cell Physiol 1989; 140 : 323-34.

16. Maher J, Colonna F, Baker D, Luzzatto L, Roberts I. Retroviral-mediated gene transfer of a mutant H-ras gene into normal human bone marrow alters myeloid cell proliferation and differentiation. Exp Hematol 1994; 22: 8-12.

17. Chabannon C, Mannoni P. Les cellules souches du sang périphérique chez l'homme. médecine/sciences $1995 ; 11$ : 17-27.

18. Gluckman E. Umbilical cord blood biology and transplantation. Curr Op Hematol $1995 ; 2$ : 413-6.

19. Gluckman E, Carosella E. Utilisation thérapeutique des cellules souches hématopoiétiques du sang de cordon. médecine/sciences $1995 ; 11: 28-34$

20. Craig W, Kay R, Cutler R. L, Lansdorp P. M. Expression of Thy-1 on human hematopoietic progenitor cells. I Exp Med 1993; 177: 1331-42.

21. Rusten LS, Jacobsen SEW, Kaalhus O, Petter O, Funderud S, Smeland EB. Functional differences between CD38- and DRsubfractions of $\mathrm{CD} 34^{+}$bone marrow cells. Blood 1994; 84: 1473-81.

22. Hatzfeld J, Panterne B, Sansilvestri P, Batard P, Lévesque J, Cardoso A, Li M, Ginsbourg M, Zhou Y, Hatzfeld A. La cellule souche hématopoiétique humaine: du mythe à la réalité. médecine/sciences 1993 ; 9: 1110-2.

23. Imbert AM, Costello R, Imbert J, Mannoni P, Bagnis C. Highly efficient retroviral gene transfer into human primary T-lymphocytes derived from the peripheral blood. Cancer Gene Ther 1994; 1: 259-65.

24. Simmons PJ, Haylock DN. Use of hematopoietic growth factors for in vitro expansion of precursor cell populations. Curr $O p$ Hematol 1995; 2 : 189-95.

25. Moore KA, Desseiroth AB, Reading CL, Williams DE, Belmont JW. Stromal support enhances cell-free retroviral vector transduction of human bone marrow long-term culture initiating cells. Blood 1992; 79: 1393-9.

26. Issaad C, Croisille L, Katz A, Vainchenker W, Coulombel L. A murine stromal cell line allows the proliferation of very primitive human $\mathrm{CD}^{2} 4^{++} / \mathrm{CD} 38^{-}$progenitor cells in long-term cultures and semisolid assays. Blood 1993; 81 : 2916-24

27. Verfaillie CM, Miller JS. A novel singlecell proliferation assay shows that long-term culture initiating cell (LTC-IC) maintenance over time results from the extensive proliferation of a small fraction of LTC-IC. Blood 1995; 86: 2137-45.

28. Mac Cune JM, Namikawa R, Kaneshima H, Shultz LD, Lieberman M, Weissman IL. The SCID-hu mouse : murine model for the analysis of human hematolymphoid differentiation and function. Science 1988; 241 : 1632-9. 